\title{
An Exploration of Various Evaluation Methods to Improve Usability of Museum Mobile Device
}

\author{
Mi-Lee Ahn, Hyun-Jin Cha, Yun-Ja Hwang, Hee-Jin Kim \\ Department of Educational Technology, Hanyang University, Seoul, 133-791
}

\begin{abstract}
Objective: This study aims towards exploring a model of the HCI evaluation methods to improve the usability of mobile device, based on a case of a Mobile PDA system in a Museum context. Background: Mobile PDA systems in a Museum context is widely utilized for the educational purposes, but it is criticized for low usability that the device only play a role in textbooks on legs without any interactive educational activities. Therefore, the usability improvements on the mobile PDA system should be considered. Method 1: This model was developed with a combination of the expert review and the user testing, and with a combination of the qualitative and quantitative studies. In more details, first of all, a qualitative study was conducted as a combination of three different methods: 1) expert review with heuristics, 2) interviews with persons working in a museum, and 3) contextual enquiry. Results 1: The experts review provided with critical usability issues, and the semi-constructive interview helped to understand the background of the mobile device. Lastly, the contextual enquiry showed user experience problems and directions of improving the device from user's perspective. Method 2: Based on the results of the qualitative study, a questionnaire was designed. Results 2: The analysis of the quantitative study was conducted to generalize the problems, and prioritize the direction of improving the device within the limitation of the cost and time in a museum. Conclusion: This study has implications in developing an example of a HCI evaluation model to improve the user experience of the mobile device as well as finding problems and directions of how to improve the mobile PDA systems in the museum. Application: In fact, most of the studies related to the evaluation of the mobile device have been conducted in a laboratory context due to the cost and time. This paper, however, attempted to apply to various HCI research techniques from different constituents in real context.
\end{abstract}

Keywords: HCI Research Methods, Mobile Device, Usability Evaluation, Museum, Contextual Enquiry

\section{Introduction}

모바일 시스템의 발전은 사회, 경제, 문화 등 거의 모든 분 야에 새로운 패러다임을 가져와 생활 방식, 소비 패턴, 관람 행위 등에서 변화를 일으키고 있다. 특히, 미술관 및 박물관 등 문화 공간에서는 교육의 활성화를 위하여 디지털 기기를 확충하고 있으며, 이를 통해 정보 습득 뿐 아니라 자칫 지루 할 수 있는 관람 문화를 좀 더 효과적이고 즐거운 경험으로
이끌 수 있는 방안을 연구하고 있다(Ahn et al., 2008b, Hall \& Bannon, 2005; Thom-Santelli et al., 2006).

하지만 대부분의 모바일 관람 기기들은 '두 다리 위의 교 과서(textbooks on legs)'라는 정체된 학습 자료 이상의 역할을 하지 못한다는 비판을 받고 있으며 (Hall \& Bannon, 2005), 관람객과 전시물 그리고 전시 정보와 관람객간의 상 호작용을 활성화시켜주고 좀 더 풍부한 전시 경험을 가지도 록 하는 역할을 제대로 수행하지 못한다는 지적을 받고 있다. 그러므로 관람객이 자연스럽게 첨단 시스템을 활용하고 필

Corresponding Author: Hyun-Jin Cha. Department of Educational Technology, Hanyang University, Seoul, 133-791.

Mobile: 010-3863-6934,E-mail: lois6934@hanmail.net

Copyright@2011 by Ergonomics Society of Korea(pISSN:1229-1684 eISSN:2093-8462). All right reserved. 
요한 정보를 습득하는 것 이상의 관람 경험을 극대화 시켜 주기 위한 매개 툴(Mediated Tool)로써의 모바일 시스템을 제공할 필요가 있다. 이를 위해선 우선 사용성 개선을 위한 적절한 방법을 적용하는 것이 필요하다(Lehn et al., 2007)

$\mathrm{HCI}$ 영역에서는 사용성을 극대화하기 위한 노력으로 다 양한 방법과 기술을 발전시켜오고 있다(Olson \& Moran, 1996). 그러나 사용 경험(UX: User eXperience)을 고려해 야 하는 많은 산업 현장에서는 어떤 방법을 어느 단계에 활 용해야 하는지에 대한 지식이 부족하고 비용과 시간의 한계 를 내세워 사용성 개선을 추진하지 못해 많은 어려움이 있다. 특히, Kjeldskov와 Graham (Ryu \& Kim, 2010)은 현재 모 바일 연구에 대한 초점이 시스템 개발 후 단순히 고전적으 로 실험실에서 프로토타입을 평가하는 것에 주로 치우쳐 있 다고 비판하였다. 그러나 모바일이라는 특성상 역동적인 사 회적 환경에서 활용된다는 점에서, 사용될 맥락속에서 평가 되는 것은 모바일 시스템의 효과 및 사용성을 이해하고 개 선하기 위해 필수적이다. 그럼에도 불구하고 모바일 산업 현 장에서 비용적, 그리고 시간적 측면을 고려했을 때, 하나의 평가 방법을 선택하는 것은 쉽지 않다.

사용성 평가 기준의 활용에 대한 많은 연구들은 적절한 평 가 방법론의 활용에 대해 논의하고 있다(Olson \& Moran, 1996; Dix et al., 1998; Preece et al., 1994, 2002; Nielsen, 1993). 각각의 평가 방법은 장점과 단점을 가지고 있기 때 문에, Nielsen(1993)은 단순히 하나의 최적의 방법을 찾아 사용성 평가를 계획하기 보다는 서로 상호보완적인 다양한 방법을 조합하여 최소의 비용으로 사용성 개선을 극대화 할 필요가 있음을 지적하고 있다.

이 연구에서는 박물관에서의 모바일 PDA 시스템 개선 사 례를 통해 모바일 기기의 사용성 평가 방법에 대하여 논의하 고자 한다. 이를 위하여 Nielsen(1993)이 추천하는 인터뷰 와 설문지의 조합과 전문가와 사용자 평가를 조합을 기반으 로 크게 정성적 평가와 정량적 평가로 나누어 사용성 평가 방법을 적용하였다.

\section{Theoretical Foundation}

\subsection{Mobile device}

최근 유비쿼터스 컴퓨팅 환경이 점차 보급되면서 모바일 기기는 인간과 주변 환경간의 인터페이스 기기로서, 시간과 장소에 제약 받지 않고, 사용자와 정보를 주고 받는 휴대용 단말기의 역할을 하고 있다. 모바일 기기는 동영상, 음성, 인 터넷 등의 멀티태스킹 작동이 가능한 기기를 의미하며, 넷북, $\mathrm{UMPC}$, 스마트폰, 아이팟(iPod), 아이폰(iPhone), 아이패드
(iPad), $\mathrm{PDA}$, 옴니아, $\mathrm{PMP}, \mathrm{MP} 3$ (동영상 재생 겸용), 전자 사전(동영상 재생 겸용), 보카마스터 등 이다(Park, 2011). 이러한 다양한 모바일 기기들은 우리 일상생활의 많은 영역 에서 점차 확대되어 사용되고 있다(Oh, 2007).

이러한 다양한 모바일 기기는 각각의 고유헌 특성이 있지 만, 공통점은 휴대성, 접근성, 상호작용성 등의 장점과 문자 입력 인터페이스 측면의 제한과 무선인터넷의 사용료에 따 른 비용의 문제점을 가지고 있다. 넷북은 기본적인 인터넷 위주의 서비스(HTLM이나 화면 기반)를 이용하는 것을 목 적으로 개발된 컴퓨터로 웹사이트 상에서의 과제 작성이나 동료간의 토론과 같은 상호작용이 용이한 장점이 있다. $\mathrm{PMP}$ 의 일종인 Apple사에서 개발한 iPod는 itunes를 통해 전 세 계에서 제공되는 오픈코스웨어 콘텐츠, Youtube를 통해 공 개되는 멀티미디어 파일 등을 다운로드 받아 언제 어디서나 쉽게 콘텐츠에 접속할 수 있고 휴대가 간편하다는 장점이 있 다(Chon \& Lee, 2011).

이러한 모바일 기기를 기능적인 측면에서 분류하면 커뮤 니케이션, 기록 및 정보 취급, 엔터테인먼트 형태로 구분할 수 있으며 그 종류는 다음 표와 같다(Park, 2011).

Table 1. Classification of mobile device according to the usage purpose(Park, M. K., 2011)

\begin{tabular}{c|l}
\hline Purpose & \multicolumn{1}{|c}{ Device } \\
\hline Communication & Mobile phone, Smart phone, PDA, Notebook \\
\hline $\begin{array}{c}\text { Records \& } \begin{array}{c}\text { information } \\
\text { handling }\end{array} \\
\text { Entertainment }\end{array}$ & $\begin{array}{l}\text { Digital camera, camcorder, electronic dictionary, } \\
\text { smart phone }\end{array}$ \\
\hline $\begin{array}{l}\text { MP3, PMP, PSP, CDP, portable cassette, } \\
\text { nintendo DS, iPod, smart phone }\end{array}$ \\
\hline
\end{tabular}

최근 $\mathrm{PDA}$ 는 기존 기능에서 벗어나 다양한 서비스를 제공 하는 형태의 멀티미디어 PDA로 변화하고 있다. 단순 PDA 는 개인 정보 관리, 게임이나 eBook 등과 같은 개별 Application을 제공하였으나 기존의 뛰어난 컴퓨팅 PDA에 무선 통신 기능이 포함된 $\mathrm{PDA}$ 폰과 단순 $\mathrm{PDA}$ 기능을 넘어 무 선 통신 기능 뿐만 아니라 휴대폰에서 개인 정보 관리, 내비 게이션 등 $(\mathrm{Kim}, 2009)$ 의 PDA의 컴퓨팅이 접목된 형태라 할 수 있는 스마트폰 등이 있다. 하지만 최근에는 PDA의 휴 대성이 강화되고 크기가 점점 작아져 스마트폰과의 구분이 모호해 지고 있다(Oh, 2009). PDA, 스마트폰 등의 모바일 기기들은 기존 $\mathrm{PC}$ 환경과는 다른 특징을 가지고 있기 때문 에 사용자 인터페이스에 관해서 고려할 필요가 있으며 최근 에 많은 연구가 되고 있다. 


\subsection{Usability evaluation methods for mobile device}

인간공학과 $\mathrm{HCI}$ 영역에서는 사람들이 알기 쉽고 사용하기 에 편리한 인터페이스를 디자인하기 위한 목적으로 사용자 중심 디자인 과정에서 다양한 평가 방법과 기술을 개발하는 데 많은 진척을 보여 왔다(Olson \& Moran, 1996). 특히, 사용자 중심 디자인 프로세스에서 인터페이스 및 제품에 대 한 평가가 단순히 한 단계에서 이루어지는 것이 아니라, 그 과정 전반에 거쳐 반복적으로 이루어질 수 있다는 점에서, 시간과 비용의 극대화를 위한 적절한 방법을 선택하는 것은 중요하다(Dix et al., 1998; Preece et al., 2002).

사용성 평가 방법론을 분류하는 방식은 평가의 기준을 어 느 관점에서 바라보느냐에 따라 학자마다 다르다. 그러므로 어떤 방법을 선택하여 사용할 것인지를 선택하기 위하여 우 선 평가 목적, 인터페이스 개발 정도, 사용자 통제 정도, 수 집 데이터 타입, 그리고 실용적인 측면에서의 상황을 고려하 여 적절한 방법을 선택해야 한다(Preece et al., 1994).

또한, Dix et al.(1998)에서는 평가 장소에 따라 실험실, 현장, 참여적 디자인(실험실과 현장을 복합적으로)으로 나누 었고, 평가 접근 방법에 따라 분석적, 리뷰적, 모델 기반 방 법으로 나누었으며, 구현된 제품을 평가하기 위한 방법으로 실험실 기반, 관찰 기반, 질문 기반 평가로 나누어, 디자인 단계, 스타일, 객관성, 측정 방법, 즉시성, 시간, 장비, 전문성 정도에 따라 방법을 선택할 수 있도록 기준을 제시하였다.

Preece et al.(1994)는 다음 Table 2처럼 평가 방법론을 선택하는 기준을 제시하고 있으며, Olson \& Moran(1996) 에서는 사용자 중심 디자인 단계에 따른 평가 방법의 선택 기준을 Table 3 와 같이 제시하였다.

평가 방법론에 대한 심층적 연구 자료와 실증적 결과에 대 한 논의에도 불구하고, 모바일 기기를 평가하기 위한 가장
Table 3. HCI methods in each step(Olson \& Moran, 1996)

\begin{tabular}{c|l}
\hline $\begin{array}{c}\text { 1. Define the } \\
\text { problem }\end{array}$ & $\begin{array}{l}\text { Naturalistic observation, interview, scenario, use } \\
\text { case, task analysis }\end{array}$ \\
\hline $\begin{array}{l}\text { 2. Generate a } \\
\text { design }\end{array}$ & $\begin{array}{l}\text { Represent conceptual model, represent interaction, } \\
\text { represent visual display, QOC, decomposition } \\
\text { analysis }\end{array}$ \\
\hline $\begin{array}{l}\text { 3. Reflect on } \\
\text { the design }\end{array}$ & $\begin{array}{l}\text { Checklists, walkthroughs, mapping analysis, } \\
\text { method analysis(GOMS, KLM etc.) }\end{array}$ \\
\hline $\begin{array}{l}\text { 4. Build a } \\
\text { prototype }\end{array}$ & Prototype tools, participatory prototyping \\
\hline $\begin{array}{l}\text { 5. Test the } \\
\text { prototype }\end{array}$ & Open testing, usability testing \\
\hline $\begin{array}{l}\text { 6. Deploy the } \\
\text { system }\end{array}$ & Internal testing, beta testing \\
\hline
\end{tabular}

적절한 하나의 방법을 선택하는 것은 쉽지 않다(Olson \& Moran, 1996). 결국, 평가하고자 하는 기기에 가장 적합한 하나의 방법이 없다는 점에서 평가 방법을 선택하는 것은 온 전히 평가자에게 달려 있다고 할 수 있다(Ryu \& Kim, 2010). 특히, 빠르게 변화하는 모바일 산업 현장에서 평가 방법을 적절하게 선택하여 활용하는 것은 사용 경험을 극대 화하고 비용적, 시간적 최소화를 위해 중요한 결정 요인이 된다. 그러므로 평가자는 하나의 방법에 의존하기 보다는 다 양한 방법을 동시에 활용함(삼각측정법)으로써 사용자의 행 위를 더 잘 이해하고 좀 더 신뢰성 있는 데이터를 수집할 수 있다(Patton, 1990). 여러 가지 방법을 혼용해서 사용하는 방법으로 Nielsen(1993)은 서로 상호보완적이면서 반복적 발견을 최소화할 수 있는 [전문가 평가(휴리스틱) 와 사용자 평가의 조합] 과 다수의 사용자로부터의 의견을 묻기 전에 소수의 사람들로부터 특정 문제를 정의하는 방식인 [오픈 인터뷰 후 설문지의 조합]을 추천하고 있다.

Table 2. HCI methods criteria(Preece et al., 1994, p696)

\begin{tabular}{|c|c|c|c|c|c|}
\hline & $\begin{array}{l}\text { Observing, monitoring } \\
\& \text { logging }\end{array}$ & $\begin{array}{l}\text { Experiment and } \\
\text { benchmark }\end{array}$ & User's opinion & Interpretive & Predictive \\
\hline Purpose & $\begin{array}{l}\text { Understanding the } \\
\text { real world }\end{array}$ & $\begin{array}{c}\text { Standards } \\
\text { Comformance }\end{array}$ & $\begin{array}{l}\text { Can be used for many } \\
\text { different purposes }\end{array}$ & $\begin{array}{l}\text { Only understanding } \\
\text { natural usage }\end{array}$ & $\begin{array}{l}\text { Engineering to } \\
\text { target }\end{array}$ \\
\hline $\begin{array}{c}\text { Interface } \\
\text { development }\end{array}$ & $\begin{array}{l}\text { Any level of } \\
\text { development }\end{array}$ & $\begin{array}{l}\text { Likely to be at least } \\
\text { working prototype }\end{array}$ & Any level of development & $\begin{array}{l}\text { Likely to be working } \\
\text { system }\end{array}$ & $\begin{array}{l}\text { Usually early at } \\
\text { mock-up stage }\end{array}$ \\
\hline $\begin{array}{c}\text { User } \\
\text { involvement }\end{array}$ & $\begin{array}{l}\text { Yes, some control of } \\
\text { tasks by users }\end{array}$ & $\begin{array}{l}\text { Yes, little or } \\
\text { no control }\end{array}$ & Yes, often no control & $\begin{array}{l}\text { Yes, Considerable } \\
\text { control }\end{array}$ & No \\
\hline Type of data & Quant./Qual. & Emphasis on Quant. & $\begin{array}{l}\text { Quant./Qual. } \\
\text { but more emphasis } \\
\text { on Quant. }\end{array}$ & Qual. & $\begin{array}{l}\text { Some Qual. but } \\
\text { emphasis on Quant. }\end{array}$ \\
\hline $\begin{array}{c}\text { Practical } \\
\text { consideration }\end{array}$ & $\begin{array}{l}\text { Special equipment } \\
\text { useful but may not be } \\
\text { essential }\end{array}$ & $\begin{array}{l}\text { Laboratory } \\
\text { conditions } \\
\text { preferred }\end{array}$ & None & $\begin{array}{l}\text { Little or no equipment } \\
\text { needed } \\
\text { Video may be used }\end{array}$ & $\begin{array}{l}\text { No equipment } \\
\text { needed }\end{array}$ \\
\hline
\end{tabular}




\section{Study 1}

이 연구에서는 박물관에서의 모바일 $\mathrm{PDA}$ 시스템 개선을 위하여 크게 정성적 평가와 정량적 평가로 나누어 사용성 평가 방법을 적용하였다. 우선 다음과 같이 정성적 평가가 시행되었다.

\subsection{Methodology: qualitative study}

현재 $\mathrm{A}$ 박물관에서 제공하고 있는 모바일 박물관 안내 시 스템의 문제점을 파악하고 사용자들의 요구 사항을 수집하 기 위하여, 다음 Table 4과 같은 절차로 정성적 연구를 수행 하였다.

Table 4. A process in the qualitative study

\begin{tabular}{c|c|c}
\hline Step & Methods & Purpose \\
\hline \multirow{2}{*}{ Step 1 } & \multirow{2}{*}{$\begin{array}{c}\text { Expert } \\
\text { review }\end{array}$} & $\begin{array}{c}\text { Initiate the usability issues } \\
\text { heuristics the problems based on the }\end{array}$ \\
\cline { 3 - 3 } Step 2 & Interview & $\begin{array}{c}\text { Understand background the system } \\
\text { of the mobile device }\end{array}$ \\
\cline { 3 - 3 } & & $\begin{array}{c}\text { Identify problems frequently issued } \\
\text { by users in reality }\end{array}$ \\
\hline \multirow{2}{*}{ Step 3 } & $\begin{array}{c}\text { Contextual } \\
\text { observation } \\
\text { \& enquiry }\end{array}$ & Usability testing in the real context \\
\cline { 3 - 3 } & & Elicit user requirements \\
\hline
\end{tabular}

1단계에서는 전문가 평가로 Nielsen(1993)이 강조한 것 처럼 기기에 대한 문제점을 파악하기 위해 사용자와 사용성 평가를 수행하기 전에 분명하게 드러나는 인터페이스적 문 제점을 파악하기 위해 제일 먼저 수행되었다. 특히 현재 박 물관에서 실제 사용되고 있는 모바일 기기가 왜 사용성 개 선이 필요한지에 대한 사용성 평가 이슈를 제기(Initiate)하 여 사용성 평가 연구를 설계하기 위한 목적으로 수행되었다. 이 평가에 참여한 전문가는 Preece et al.(2007)에서 정의 된 것처럼 $\mathrm{HCI}$ 에 background를 가지고 있고 사용성 평가 방법을 알고 있는 연구자 ${ }^{1}$ 4인(Nielsen, 2005)으로 구성하 여 Nielsen, 1994)에서 제시한 Heuristics에 원칙을 근거하 여 분석적 평가를 실시하였다.

2단계에서는 박물관에서 PDA를 제공하는 주주(Stakeholder) 측 관계자인 사업기획팀장, 경영관리부 팀장, 고객지 원팀 담당자, $\mathrm{PDA}$ 대여소 팀장 등과 반구조화 인터뷰를 실

${ }^{1}$ 전문가는 $\mathrm{HCI}$ 과목을 이수하고 관련 프로젝트를 진행하여 사용성 평가 방법에 대한 지식을 가지고 있는 석사 이상의 4 인으로 구성함.
시하여 그 모바일 기기 도입 배경, 설계 및 유지보수 문제 등에 관한 인터뷰를 실시하여, 사용성 개선이 좀 더 현실적 인 문맥 내에서 일어날 수 있도록 관련 배경을 조사하였다. 마지막으로 1 단계와 2단계에서 축적된 지식을 바탕으로 문맥적 관찰 및 질의(Contextual Observation \& Enquiry) 연구를 설계하였다. 박물관의 주요 관람객 층인 초등학교 3 학년에서 중학교 1 학년 4 명에 대해 사전 인터뷰하고, 박물 관 관람 중 학습지를 가지고 Task를 수행하면서 자신의 생 각을 Think-aloud하고, 사후 인터뷰를 진행하는 방식으로 문맥적 관찰 및 질의 연구를 수행하였다(Ahn et al., 2008a).

\subsection{Result of the qualitative study}

본 연구는 Table 5 와 같이 3 단계에 걸쳐 수집된 정성 적 자료를 분석하였다. 우선 1 단계에서는 전문가들은 각자 Heuristic 원칙에 따라 사용자 인터페이스를 평가하고 문제 점을 기술한 후 이를 모아 논의를 통해 관련 문제를 리스트 화했다(Nielsen, 2005). 2단계에서는 모바일 기기에 대한 배경 지식을 가지는 목적에 더하여 대여소 팀장과의 인터뷰 에서 얻은 사용자들이 제기한 사용성 문제에 관한 내용을 모두 전사한 후 "Critical Incident Technique"을 활용하여 Critical Breakdowns \& Incidents를 중심으로 Pattern을 확인하고 목록화하여 문제점을 찾아 기술하였다(Preece et al., 2007). 또한 3단계에 수행한 Contextual Enquiry는 기 호학적 상호작용 관점에서 분석하였다(Ahn et al., 2008a). 이 세 단계로부터 수집된 데이터를 모두 수합하여 Category 화 했고 최종적인 분석은 분석자 삼각측정법(Patton, 1990) 을 활용하여 다양한 분석자의 시각에서 데이터를 분석하여 정성적 데이터의 타당성을 높이는 방법으로, 이 연구를 수행 하는 4 인의 연구자들이 제각기 관련 자료를 검토하고 함께 토론하는 과정을 통해 최종의 결과를 도출하였다.

Table 5. A process in the qualitative study

\begin{tabular}{c|c|c}
\hline Step & Methods & Analysis method \\
\hline Step 1 & $\begin{array}{c}\text { Expert } \\
\text { review }\end{array}$ & $\begin{array}{c}\text { Personal notes of each expert }-> \\
\text { Aggregation }->\text { Discussion }-> \\
\text { Usability Problems Lists(Nielsen, 2005) }\end{array}$ \\
\hline Step 2 & Interview & $\begin{array}{c}\text { Critical incident technique } \\
\text { (Preece et al., 2007) }\end{array}$ \\
\hline Step 3 & $\begin{array}{c}\text { Contextual } \\
\text { observation } \\
\text { \& enquiry }\end{array}$ & $\begin{array}{c}\text { Content analysis based on symbolic } \\
\text { interactionism \& analysts triangulation } \\
\text { (Patton, 1990) }\end{array}$ \\
\hline
\end{tabular}

위의 분석 결과로 정리된 Category를 종합하고 각 카테 고리에 따라 제기된 문제점을 Table 6와 같이 크게 네 가지 
영역으로 나눌 수 있다. Table 6의 4개 영역을 통해 제기된 사용성 문제점은 우선 하드웨어적 측면에서는 $\mathrm{PDA}$ 크기가 너무 크고 무거우며, 로딩하는데 속도가 너무 느리다고 지적 되고 있다. 다음은 사용자 인터페이스의 자동/수동 변환 시 불편함, 버튼 사이즈와 위치의 불편함을 지적하고 있다. 그 리고 내용적 측면은 박물관 유물에 대한 설명의 양이 적음, 텍스트 읽기의 어려움, 설명이 없는 것으로 인한 불편함을 지적하고 있다. 마지막으로 경험적인 면에서 $\mathrm{PDA}$ 를 활용하 여 다른 사용자와의 상호작용을 하거나 다른 인터엑션이 없 다는 것과 혼자 하는 관람해야 하는 불만과 이어폰 착용감 등을 지적하였다.

Table 6. Result of the qualitative study 1

\begin{tabular}{c|l}
\hline Category & \multicolumn{1}{|c}{ Usability issues } \\
\hline $\begin{array}{c}\text { Hardware } \\
\text { (HW) }\end{array}$ & $\begin{array}{l}\text { PDA size, weight, shape, loading speed, } \\
\text { battery }\end{array}$ \\
\hline $\begin{array}{c}\text { User interface } \\
\text { (UI) }\end{array}$ & $\begin{array}{l}\text { Auto/Manual transfer, size and location of } \\
\text { buttons, size of font and image, display space } \\
\text { design, controller of audio, absence of } \\
\text { necessary functionality, inefficient search } \\
\text { engine, unusable interface }\end{array}$ \\
\hline Contents & $\begin{array}{l}\text { Quantity in texts and images, no text and } \\
\text { explanation about some relics, preference of } \\
\text { voice, the number of foreign language, too } \\
\text { simple explanation without any emphasis }\end{array}$ \\
\hline $\begin{array}{c}\text { Experience } \\
\text { (UX) }\end{array}$ & $\begin{array}{l}\text { Experience using it alone, feeling to put on } \\
\text { earphone, expensive cost for borrowing the } \\
\text { PDA }\end{array}$ \\
\hline
\end{tabular}

\subsection{Implication of study 1}

본 연구에서 적용하고 있는 3 단계에 거친 정성적 평가 방 법론은 결론을 도출하기까지 각 방법론이 가지는 장점을 극 대화 하고 있다. 예를 들어, 전문가 평가에서는 하드웨어적, 인터페이스적, 콘텐츠적인 사용성 이슈를 개괄적으로 분류하 고 찾아낼 수 있었지만, 실제 문맥에서의 사용자 테스트는 직접 사용자가 부딪히는 문제와 그에 대한 경험적 코멘트를 바탕으로 좀 더 깊이 있는 문제점을 파악할 수 있었다. 특히 오동작이라든지 경험적 측면에서의 코멘트로부터 <사용자 경험>이라는 새로운 Category를 도출할 수 있었다. 관계자 와의 인터뷰를 통해서는 모바일 기기의 주요 사용자층에 대 한 배경, 유지보수 등 기기 도입 배경, 경제적인 이슈, 유물 업데이트 등 콘텐츠적인 측면 등에 대한 문제점을 파악할 수 있었다.

특히, 사용자들과의 사후 인터뷰에서 PDA 시스템을 어떻 게 바꾸어야 할지 논의한 결과 닌텐도처럼 듀얼 모니터 형식
으로 위쪽에서는 정보를 아래쪽에서는 메모를 할 수 있는 형 태, 네비게이션 기능을 가진 세로 형태의 바 기기 등 혁신적 인 하드웨어 형태를 제안하였고, $3 \mathrm{D}$ 동영상으로 유물을 관 찰하거나 Task로 주어진 학습지를 포함한 기기 등 콘텐츠 에 대한 아이디어를 제안하여, 오픈 인터뷰는 좀 더 자유로 운 의견 교환을 통해 혁신적인 디자인 모델에 대한 논의가 가능하다는 것을 보여주었다.

이처럼 세 가지의 다른 사용성 평가 방법은 모바일 기기를 평가하는데 각각의 목적에 따라 다른 관점과 이슈를 제기하 여 더 많은 사용성 문제를 찾고, 개선점을 도출하도록 시사 점을 주었다.

\section{Study 2}

\subsection{Methodology: quantitative study}

다수의 의견은 정성적 데이터를 일반화(generalize)하고, 현재 박물관에서 활용하는 시스템에 대하여 사용성 개선을 요구할 수 있는 근거가 된다. 이를 위해 설문조사를 실시하 였고, 정성적 연구 분석 결과로부터 최종적으로 도출된 사용 성 문제점을 바탕으로 각 문제점을 다시 확인하였다. 문제의 심각성 (Severity)을 계산하며, 개선 방향을 도출할 수 있도 록 다음 Table 7 과 같이 Study 2를 설계하였다.

설문지의 각 문항은 영역에 따라 감정/느낌, 만족도 등을 표기하도록 하였다. 사용자 경험에 대해서는 어려움, 짜증남, 흥미 없음, 자랑스럽다 등의 사람의 감정을 표현하도록 하였 으며, 다른 영역에 대해서는 만족 여부를 5 단계의 스케일로 체크하게 하였다. 35-36번은 PDA에 추가하고 싶은 기능 5 가지를 체크하게 하였다. 정성적 연구에서 주로 언급된 항 목을 선택항목으로 나열함으로써 설문자들이 쉽게 체크하도

Table 7. Design of the questionnaire 1

\begin{tabular}{c|c|c}
\hline Questions & Contents & Category \\
\hline $1 \sim 8$ & User profiles & \\
\hline $9 \sim 13$ & $\begin{array}{c}\text { Overall user experience satisfaction } \\
\text { of PDA usage }\end{array}$ & UX \\
\hline $14 \sim 19$ & $\begin{array}{c}\text { Satisfaction of PDA user interface } \\
\text { and menu }\end{array}$ & UI \\
\hline $20 \sim 26$ & Satisfaction of PDA hardware & HW \\
\hline $27 \sim 30$ & Satisfaction of PDA content & Content \\
\hline $31 \sim 34$ & Usage value & UX \\
\hline $35 \sim 36$ & Additional functionalities & UI \\
\hline $37 \sim 38$ & $\begin{array}{c}\text { Good experience \& } \\
\text { bad experience(Open Answer) }\end{array}$ & UX \\
\hline
\end{tabular}


록 하였고, 이를 기반으로 빈도수를 계산하였다. 그리고 설 문지의 마지막 항목인 37-38번에서는 가장 짜증나게 한 점 과 가장 좋았던 점을 주관식으로 서술하도록 하였다.

\subsection{Results of the quantitative study}

총 65 부의 설문지를 수집하였고, SPSS 14.0 를 활용해 기 술 통계 분석, 빈도 분석, 교차 분석을 실시하였다. 설문 분 석 결과 다음 Table 8과 같이 모바일 안내 시스템에서 제공 하고 있는 기능에서, 자주 사용하지 않는 메뉴에 대한 정보 와 사용자 만족도 정도를 알 수 있다. 즉, 모바일 기기를 활 용한 관람에서 전혀 사용하지 않는 기능은 북마크, 추천코스, 검색 등의 순이었으며 만족도에서는 추천코스, 위치찾기, 게 임 순으로 나타났다.

또한 하드웨어 측면과 콘텐츠 측면에서는 이어폰 착용감, 무게, 로딩 속도, 버튼 모양, 설명의 양 순으로 불만족도가 높았다. 이처럼 정량적 연구로부터의 통계 수치는 정성적 연구를 통해 드러나 다양한 문제점들에서 사용성 문제의 강도(Severity)를 비교하여 개선 사항 제시 시 우선 순위 (Priority)를 정할 수 있는 기초 데이터를 제공해 주었다.

Table 8. Result of the questionnaire

\begin{tabular}{|c|c|c|}
\hline & Never used & Dissatisfaction level \\
\hline Search & $31.3 \%$ & $20.6 \%$ \\
\hline Location & $27.9 \%$ & $28 \%$ \\
\hline Bookmark & $38.8 \%$ & $25.4 \%$ \\
\hline Help & $32.8 \%$ & $25.4 \%$ \\
\hline Treasure-hunt game & $20.9 \%$ & $26.8 \%$ \\
\hline \multirow[t]{2}{*}{ Recommended course } & $38.2 \%$ & $29.4 \%$ \\
\hline & \multicolumn{2}{|c|}{ Dissatisfaction level } \\
\hline Shape & \multicolumn{2}{|r|}{$19.4 \%$} \\
\hline Weight & \multicolumn{2}{|r|}{$57.4 \%$} \\
\hline Size & \multicolumn{2}{|r|}{$10.6 \%$} \\
\hline Button shape & \multicolumn{2}{|r|}{$28.4 \%$} \\
\hline Font size & \multicolumn{2}{|r|}{$26.5 \%$} \\
\hline Earphone & \multicolumn{2}{|r|}{$69.1 \%$} \\
\hline Loading time & \multicolumn{2}{|r|}{$32.4 \%$} \\
\hline Image & \multicolumn{2}{|r|}{$7.4 \%$} \\
\hline Voice & \multicolumn{2}{|r|}{$20.6 \%$} \\
\hline Amount of text & \multicolumn{2}{|r|}{$22.1 \%$} \\
\hline Explanation & \multicolumn{2}{|r|}{$10.4 \%$} \\
\hline
\end{tabular}

교차 분석에 의한 결과는 [사용의 어려움] 과 [PDA 사용
경험]사이에 그리고 [PDA 사용 경험] 은 [PDA의 도움 정 도] 에 유의미한 차이를 보여주어, $\mathrm{PDA}$ 가 어렵게 느낀 사용 자는 사용 경험 또한 낮았고 $\left(\chi^{2}=17,22, p<0.05\right)$, 사용 경 험이 안좋다고 느낀 사용자는 $\mathrm{PDA}$ 가 도움이 안된다고 생각 하는 것으로 드러났다 $\left(\chi^{2}=77,64, p<0.05\right)$. 결국 사용성은 사용 경험과 연결되고 사용 경험은 기기의 필요 여부를 결 정하는 중요한 요인이 됨을 양적 연구를 통해 볼 수 있었 다. 또한 사용자의 기기 친숙도 여부에 따라 PDA 하드웨 어 및 기능을 평가하는 정도가 달랐다. 전문가급의 사용자 는 무게 $\left(\chi^{2}=18,42, p<0.05\right)$, 화면의 그림/사진 $\left(\chi^{2}=18,66\right.$, $p<0.05)$, 설명의 양 $\left(\chi^{2}=16,45, p<0.05\right)$, 보고 있는 유물 설명 $\left(\chi^{2}=14,03, p<0.05\right)$ 에 대한 만족도가 훨씬 더 낮은 것을 보여줌으로써 사용자의 기기 사용 능력에 따라 기기에 대한 높은 기대치를 가지고 있는 것으로 드러났다. 이는 사 용자 층별로 요구 사항이 다를 수 있음을 보여주었다.

개선 사항을 도출하기 위해 추가 기능에 대해 빈도 분석 한 결과 1 5위는 다음 Table 9 과 같이 도출되었다.

Table 9. Preference for new feature(Multiple Answers)

\begin{tabular}{c|c|c}
\hline Rank & New feature & Frequency \\
\hline 1 & Bring the favorite relic information to home & 44 \\
\hline 2 & Navigation & 40 \\
\hline 3 & Video Clip & 37 \\
\hline 4 & Internet(Search \& email) & 33 \\
\hline 5 & Interesting learning material \& game & 28 \\
\hline 5 & Digital camera & 28 \\
\hline
\end{tabular}

또한 37번 문항에 '가장 짜증나게 한 요소들'에 대한 결 과는 1 위 오동작, 2 위 무거움, 3 위/4위 이어폰과 로딩시간, 5 위 목소리 등으로 정성적 연구와 비슷한 결과를 보여주었 다. 또한, 설문지 결과로부터 눈여겨보아야 할 점은 사용자 들이 "내가 좋아하는 유물 자료 가져가기"를 가장 원하는 기 능으로 보고 있지만 Table 8처럼 "북마크" 기능은 전혀 사 용하지 않는 사용자가 약 $39 \%$ 나 되면서, 불만족도도 높다 는 점에서 개인화(Personalization)에 대한 요구 사항을 어 떻게 개선해 줄 지에 대한 연구가 필요함을 알 수 있었다.

\section{Discussion}

이 연구로부터 모바일 기기의 사용성 평가에 대하여 다양 한 방법론적인 시사점을 얻을 수 있었다. 우선 첫 번째로 정 성적 연구 방법(전문가 리뷰, 관계자 인터뷰, 문맥적 관찰) 
의 조합을 통해, 데이터를 수집함으로써 정성적 데이터의 다 원화(Triangulation)를 시도하였다는 점이다. 이는 Table 4 에서 제시한 것처럼 각 단계에서 필요한 평가 목적과 내용을 반영하고 있어, 모바일 기기를 평가하는데 다른 관점에서 문 제점을 찾아내어, 좀 더 실용적으로 사용자 중심의 분석이 가 능하게 하였다는 점이다. 대부분 정성적 연구라고 하면 민족 지학상의(Ethnographic) 또는 인류학적(Anthropological) 연구를 떠올려 많은 시간과 노력이 필요한 것으로 간주하여 도입하기 어렵게 느낀다. 하지만 목적에 따라 초점을 맞춰 데이터를 분석할 수 있도록 현장에서 비교적 쉽고 빠르게 적용할 수 있는 너무 정교하지 않은(coarse-grained) 정 성적 연구 방법(Preece et al., 2002)인 Critical Incident Technique 등을 선택함으로써, 시간과 비용의 극대화를 가 져올 수 있다. 대신 다양한 정성적 연구 방법의 조합을 통해 관점을 다원화(Triangulation) 하여 데이터의 신뢰를 확보할 수 있다.

두 번째로 정량적 연구를 통해 정성적 연구의 데이터를 일 반화(Generalize)하고 문제의 강도(Severity)를 측정하여, 개선 사항 고려 시 우선 순위(Prioritize)와 중요도를 정할 수 있는 기준을 마련하였다는 점이다. 현 박물관의 경제적 상황을 고려할 때, 분석된 모든 문제점을 개선하기에는 시간 적, 비용적으로 쉽지 않다. 이런 상황에서 정량적 연구는 어 떤 부분에 초점을 맞추어 개선해야 할지 결정할 수 있는 근 거 데이터가 된다. 또한 앞서 설명한 것처럼 정성적 연구 결과의 오픈 인터뷰로부터 얻은 개선 사항에 대한 결과가 너무 이상적이 될 수 있다는 점에서, 사용자가 원하는 추가 설계나 기능을 모두 반영할 수 없다. 그러므로, 현장에서는 다수의 사용자가 원하는 사항으로 trade-off하여 결정하고 (Decision-making) 디자인에 반영함으로써 이에 대한 적 절한 이론적 근거(Rationale)를 제시할 수 있다.

이 연구의 마지막 함의로는, 모바일 기기를 평가할 때 네 가지 평가 기준 영역으로 하드웨어적 측면, 인터페이스적인 측면, 콘텐츠 측면, 그리고 사용자 경험 측면 요소를 추출하 였다는 점이다. 이는 다양한 정성적 연구의 조합을 통해 모 바일 기기에서 제기될 수 있는 문제점 영역을 분류한 결과 이 네 가지 측면에서 사용성 문제를 제기하였고, 이에 대한 설문지 설계를 통해 이 네 가지 영역에서 사용성을 개선하는 방향으로 연구가 진행되었다. 특히 실험실 기반이나 전문가 평가에서는 <사용 경험> 측면이 쉽게 간과될 수 있는 영역 이므로 사용자와의 평가를 통해 이에 대한 심층적인 고려가 필요하다.

이러한 시사점에 덧붙여, 박물관이라는 현장에서 실제 초 등학생인 사용자와 사용성 평가(Contextual Enquiry)를 진 행하면서 현장 연구에서 고려되어야 하는 시사점도 도출되 었다(Ahn et al., 2008a). 초등학생을 대상으로 했기 때문에
실험 상황에서 다른 친구들과 이야기를 하는 등의 돌발적인 행동을 통제하고, 어른들도 어려운 Think-aloud를 지속적 으로 할 수 있도록 격려해야 하는 어려움이 있었다. 이러한 현장 연구의 문제점을 고찰하면서 향후 모바일 기기의 현장 연구가 좀 더 정교한 방향으로 이루어지기 위해서는 다양한 모바일 기기에 따른 현장 연구의 차이점을 논의하고 각각 모바일 기기의 특성에 따른 평가 모델에 대한 연구가 이루어 져야 한다. 또한 향후 연구에 대한 제안으로 이 연구에서는 일반적으로 활용되는 Nielsen의 Heuristics을 적용하였지만, 모바일 기기에 특성화하여 평가할 수 있는 네 가지 영역에서 의 휴리스틱 기준을 마련하여 박물관 등에 제공되는 모바일 안내 시스템 설계 및 개선 시 사용성 평가 기준으로 마련하 여 적용할 수 있을 것이다.

\section{Conclusion}

이 연구에서는 박물관에서의 모바일 안내 PDA 시스템 개 선 사례를 통해 모바일 기기의 사용성 평가 방법을 탐구하였 다. 이를 위해 다양한 사용성 평가 방법에 대한 선택 기준과 방법론에 대한 선행 연구를 바탕으로 다음 Figure 1과 같이 평가 모델을 설계하여 시행하였고, 하드웨어적, 인터페이스, 콘텐츠, 그리고 사용 경험 측면의 영역에서의 문제점과 개선 방향을 도출하였다.

각각의 사용성 평가 방법은 고유의 특징과 장 - 단점을 가 지고 있다는 점에서 어떤 상황에서 어떤 목적으로 사용할 것 인지에 따라서 적절히 계획하여 시행할 필요가 있다. 그러므

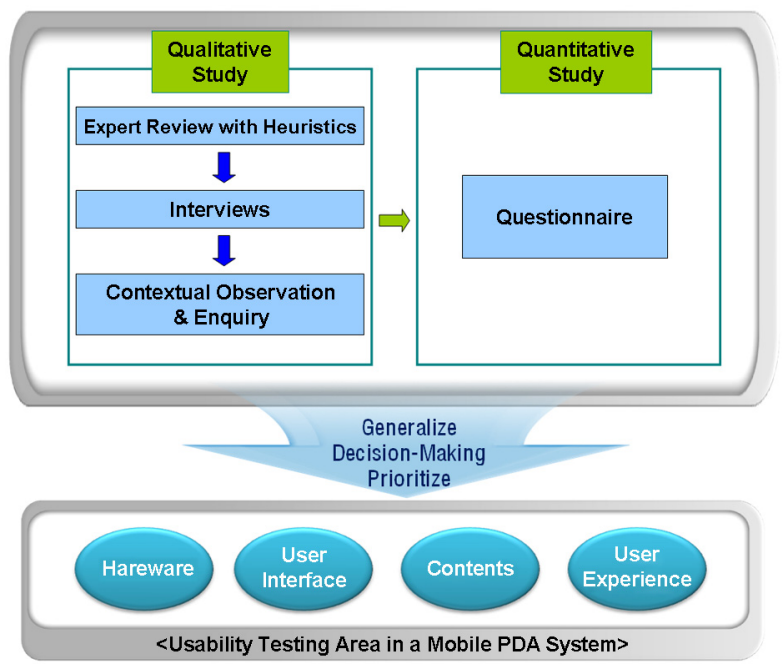

Figure 1. A Model of HCI evaluation methods for the usability of mobile device 
로 이 연구에서는 단순히 한 가지 방법에 의존하기 보다는 다양한 정성적 연구 방법을 조합하여 활용하였다. 우선 시스 템의 완전한 이해를 통해 분명하고 확실한 사용성 이슈를 파악하고 문제를 제기하기 위해 [휴리스틱에 근거한 전문가 평가]를 실시하였다. 2 단계로 그 기기를 도입한 배경과 다년 간 활용되면서 변화해 온 역사, 맥락 등을 파악하기 위해 [관 계자 인터뷰]를, 그리고 3단계로 앞서 진행된 결과를 바탕으 로 최종적으로 사용자로부터 현장에서의 문제점을 파악하고 개선 방향을 도출하기 위한 [문맥적 관찰 및 질의]가 진행되 었다. 이 정성적 연구로부터 나온 데이터를 바탕으로 설계 된 설문지를 통해 분석 데이터를 일반화(Generalizae)하고 문제점의 강도(Severity)를 측정하여 개선 사항의 우선 순 위(Priority)를 정하기 위하여 정량적 연구가 시행되었다.

이 연구는 $\mathrm{A}$ 박물관의 모바일 기기의 사용성 개선 사항 도출하였고, 사용성은 사용 경험과 연결되고 사용 경험은 기 기의 필요 여부를 결정하는 중요한 요인이 된다는 점, 그리 고 사용자 층별로 기기에 대한 기대치가 다르다는 점 등 모 바일 기기에 대한 다양한 시사점을 도출하였다.

또한, 앞으로 유비쿼터스 시대를 맞아 사회, 경제, 교육, 문화 등 각 분야에 도입될 모바일 기기의 사용성 평가를 위 한 모델을 구축하였다는 점에서 의의가 있다. 특히, 이 모델 은 산업 현장에서 단순히 실험실 환경의 평가만을 수행하는 것이 아니라, 실제 문맥으로부터 정성적 평가를 쉽고 경제적 으로 수행할 수 있는 모델을 보여주었다. 이는 경제성뿐 아 니라, 다양한 관점에서의 데이터를 통해 문제점 및 개선 사 항을 추출하고 이를 일반화(Generalize)하여, Trade-off를 결정할 수 있는 합리적 근거(Rationale)를 마련하는 실용적 인 모델로 활용될 수 있을 것이다.

\section{References}

Ahn, M. L., et al. "Direction of Improving Mobile System to Enhance a Learning Experience", Proceeding of Korean Society for Educational Technology, Seoul, Korea, 2008a.

Ahn, M. L., et al. "User Centered Design and Development Strategies for Participatory Learning Media", Proceeding of HCI Korea, Kangwon, Korea, 2008b.

Chon, E. H. \& Lee, Y. M., "The Analysis on Teaching and Learning Activities Using Mobile Devices in Higher Education", The Korea Contents Association, Seoul, Korea, 11(2), 477-486, 2011.

Dix, A., et al. Human Computer Interaction, 2nd ed. Prentice Hall, 1998.

Hall, T. \& Bannon, L. "Designing Ubiquitous Computing to Enhance Children's Interaction in Museums", IDC 2005, USA, 2005.

Kim, H. M., "Study on mobile digital text-book design for the vitalization educational function of museum", Hanyang Univ. Master's Thesis,
2009.

Lehn, D., et al. "Engaging Constable: Revealing Art with New Technology", CHI2007 Proceedings, USA, 2007.

Nielsen, J., Usability Engineering, Academic Press. 1993.

Nielsen, J., How to conduct a heuristic evaluation. Jakob Nielsen's Alertbox, 2005, http://www.useit.com/papers/heuristic/heuristic_evaluation.html (Retrieved Septermber 16, 2011).

Oh, H. Y., "Soft Keyboard Interface Design for Mobile Device", The Korea Contents Association, Seoul, Korea, 7(6), 79-88, 2007.

Oh, M., "A Study of PDA Phone Interface Design", Ewha Womans Univ. Master Thesis, 2009.

Olson, J. \& Moran, T., Mapping the method muddle: Guidance in using methods for user interface design. In M. Rudisill, C. Lewis, P. Polson and T. McKay (eds) Human-Computer Interface Design: Success Stories, Emerging Methods, and Real-World Context, MorganKaufmann, 269-300, 1996.

Park, M. K., "The Study of the Way for Learning Application using Mobile Devices", Incheon Univ., Incheon, Korea, 2011.

Park, Y. S., et al. "Heuristic Evaluation Items for Investigating Usability of a Mobile Phone UI", Proceeding of 2008 Spring Conference of Ergonomics Society of Korea, Kumi, Korea, 2008.

Patton, M. Q., Qualitative Evaluation and Research Methods, 2nd ed. SAGE Publications, 1990.

Preece, J., et. al. Human Computer Interaction, Addison-Wesley, 1994.

Preece, J., et al. Interaction Design, John Wiley \& Sons, 2002.

Preece, J., et al. Interaction Design, John Wiley \& Sons, 2007.

Ryu, J. H. \& Kim, M. J., Understanding Mobile Human-Computer Interaction, HakJiSa, 2010.

Thom-Santelli J., et al. "Beyond Just the Facts: Transforming the Museum Learning Experience", CHI 2006, Canada, 2006.

Mi-Lee Ahn: mlahn@hanyang.ac.kr

Highest degree: $\mathrm{PhD}$, Department of Educational Technology, Purdue University

Position title: Associate Professor, Department of Educational

Technology, Hanyang University

Areas of interest: e-Learning Accessibility, HCI, Learner Experience,

Universal Design for Learning, Global Education

Hyun-Jin Cha: lois6934@hanmail.net

Highest degree: $\mathrm{PhD}$. Candidate, Department of Educational Technology, Hanyang University

Position title: Researcher, KERIS

Areas of interest: ICT use in education, HCI, UDL (Universal Design for Learning), Ubiquitous Computing in Education 
Yun-Ja Hwang: yjhwang@hanyang.ac.kr

Highest degree: $\mathrm{PhD}$. Candidate, Department of Educational Technology, Hanyang University

Areas of interest: Computer Education, HCI, Universal Design for Learning (UDL), e-Learning

Heejin Kim: khj0510@hanyang.ac.kr

Highest degree: $\mathrm{PhD}$. Student, Department of Educational Technology, Hanyang University

Areas of interest: Universal Design for Learning, Human Computer

Interaction, Mobile Learning, Computer Education
Date Received : 2011-07-07

Date Revised :2011-11-08

Date Accepted : 2011-11-10 